\title{
COMPASS results on the transverse spin asymmetry in hadron-pair production in SIDIS
}

\author{
Christopher Braun ${ }^{1, a}$ \\ ${ }^{1}$ on behalf of the COMPASS Collaboration \\ Physikalisches Institut IV of the University Erlangen-Nürnberg (FAU) \\ Erwin-Rommel-Strasse 1, 91058 Erlangen, Germany
}

\begin{abstract}
The parton distribution function $h_{1}^{q}(x)$ of a transversely polarized quark $q$ inside a transversely polarized nucleon, is chiral-odd and therefore not accessible in inclusive deep-inelastic scattering. It can however be observed in semi-inclusive deep-inelastic scattering (SIDIS) in combination with another chiral-odd function, for instance the dihadron interference fragmentation function (DiFF) $H_{1}^{\triangleleft q}$. The $160 \mathrm{GeV} / c$ polarized muon beam of CERN's M2 beamline allows COMPASS to investigate transverse spin effects using polarized solidstate targets. In this contribution an overview of COMPASS results for the azimuthal asymmetry in identified dihadron production is given. Pions and kaons were identified by a RICH detector. Recently, the full set of this asymmetry from the COMPASS data on the deuteron and the proton target became available. All data, including early data sets, were analyzed using the same methods and requirements. The results are compared to model predictions and HERMES data. Also a point-by-point extraction of the Transversity distribution functions $h_{1}(x)$ for $u$ and $d$ quarks was carried out with this full data set. Further investigations on the correlation between the dihadron asymmetry and the Collins asymmetries of the two hadrons of the pair have revealed an interesting dependence of these three asymmetries on the difference $\Delta \Phi$ of the azimuthal angles of the two hadrons.
\end{abstract}

\section{Theoretical framework and data selection}

In the SIDIS process $\mu N \rightarrow \mu^{\prime} h_{1} h_{2} X$ the incoming lepton is scattered off a transversely polarized quark inside the nucleon via the exchange of a virtual photon. The struck quark hadronizes into at least two unpolarized hadrons. For each oppositely charged hadron-pair, the quantity $\boldsymbol{R}$ is defined, i.e. their normalized relative momentum. Figure 1 shows a simplified scheme of this process. In the SIDIS cross section the angle $\phi_{R}$ between the dihadron plane and the scattering plane and the azimuthal angle of the spin of the initial quark $\phi_{S}$ appear in an azimuthal modulation as a function of $\phi_{R S}=\phi_{R}+\phi_{S}-\pi[1,2]$.

To select DIS events in general, kinematic cuts on the negative square of the four-momentum transfer $Q^{2}>$ $1(\mathrm{GeV} / c)^{2}$, the fractional energy transfer of the muon $0.1<y<0.9$ and the hadronic invariant mass $W>$ $5 \mathrm{GeV} / c^{2}$ were applied. The hadron-pair sample requires more selection w.r.t. the single hadron asymmetries analysis [3], of which the requirement for a vertex with at least three outgoing tracks (scattered $\mu^{+}$and two hadrons) is the most fundamental one. All possible combinations of oppositely charged hadron-pairs originating from the vertex are taken into account in the analysis. Each of these hadrons has to have a fractional energy $z>0.1$ and a Feynman

\footnotetext{
ae-mail: christopher.braun@cern.ch
}

$x_{F}>0.1$, to ensure that the hadrons are not produced in target fragmentation. Exclusively produced $\rho$ mesons are rejected by a cut on the missing energy $E_{\text {miss }}>3 \mathrm{GeV}$ of the hadron-pair. Finally a cut of $R_{T}>0.07 \mathrm{GeV} / c$ ensures a well defined azimuthal angle $\phi_{R}$. After all cuts the full statistics on the proton target consists of $45.5 \times 10^{6} h^{+} h^{-}$ pairs, of which $28.0 \times 10^{6}$ are identified as pion-pairs. The deuteron sample consists of $4.0 \times 10^{6}$ pion-pairs out of $6.5 \times 10^{6} h^{+} h^{-}$pairs.

\section{Results from the 2003-04 deuteron data}

The dihadron asymmetry of pion-pairs $\pi^{+} \pi^{-}$for the data collected in 2003-04 on the deuteron target are consistently small and compatible with zero within the uncertainties, see Fig. 2. Furthermore no specific trend is visible for their dependence on $x, z$ and the invariant mass of the dihadron system $M_{i n v}$. This result is in line with the COMPASS measurement of the Collins asymmetry on the deuteron [4] and the all-hadron $h^{+} h^{-}$results in [5]. This is interpreted as the consequence of a close to complete cancellation of the $u$ and $d$ quark contributions to the total Transversity distribution of the deuteron, which is also predicted by the available models $[6,7]$. The asymmetries of the other identified pair combinations $K^{+} K^{-}, \pi^{+} K^{-}$and $K^{+} \pi^{-}$are compatible with zero within their uncertainties or give only weak hints of nonzero signals. 


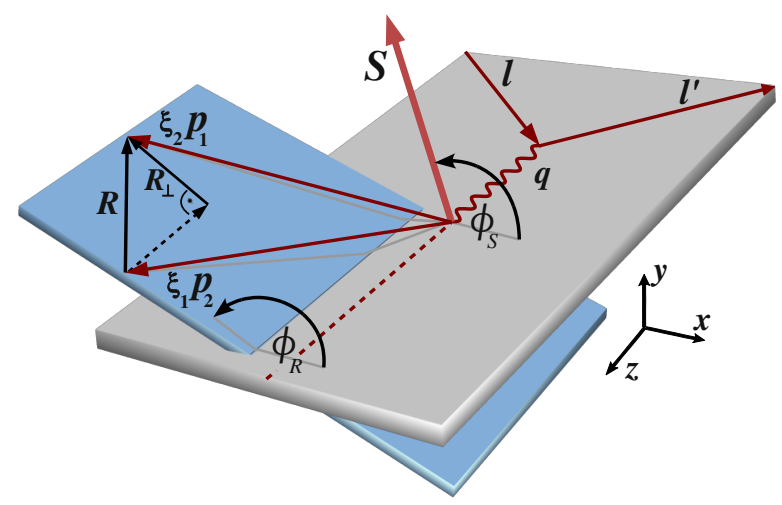

Figure 1. Simplified scheme of the dihadron production process: The incoming lepton and scattered lepton with their threemomenta $\boldsymbol{l}$ and $\boldsymbol{l}^{\prime}$ define the scattering plane (gray). The threemomentum of the virtual photon is denoted by $\boldsymbol{q}$. The angle $\phi_{S}$ is the azimuthal angle of the spin $S$ of the fragmenting quark. Each hadron $i$ has its three-momentum $\boldsymbol{p}_{i}$, together they define the hadron plane (blue). The corresponding $\xi_{i}$ values are used for a normalization of the difference vector $\boldsymbol{R}$, i.e. $\boldsymbol{R}=\left(z_{2} \boldsymbol{p}_{1}-z_{1} \boldsymbol{p}_{2}\right) /\left(z_{1}+z_{2}\right)=\xi_{2} \boldsymbol{p}_{1}-\xi_{1} \boldsymbol{p}_{2}$. Hence $\phi_{R}$ is the azimuthal angle of $\boldsymbol{R}$ and $\boldsymbol{R}_{T}$ is its component perpendicular to $\boldsymbol{q}$.

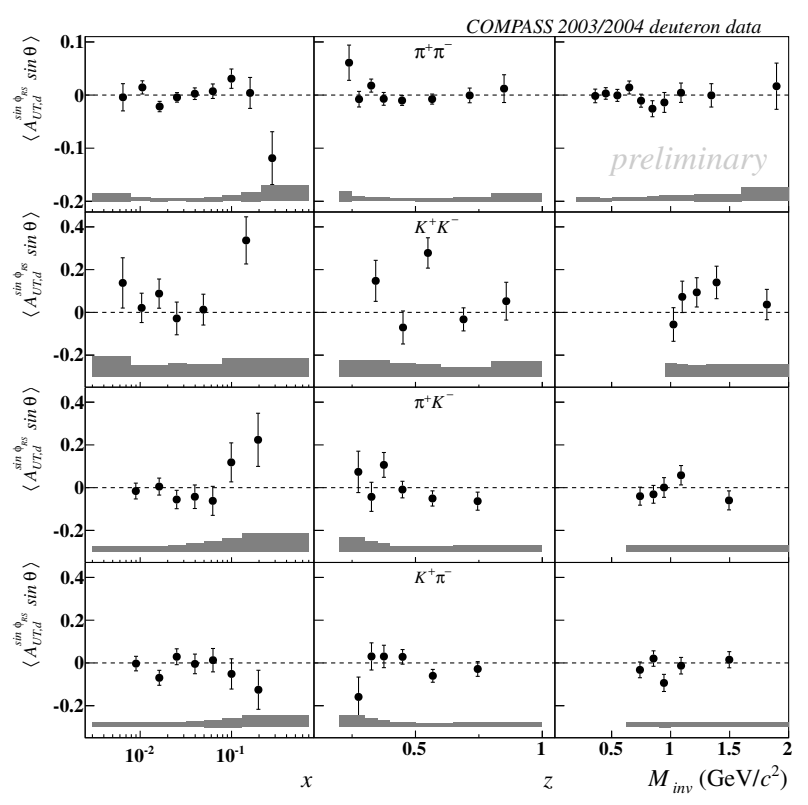

Figure 2. The identified dihadron asymmetries from the 2003-04 deuteron data [8].

\section{Results from the combined 2007/2010 proton data}

The first measurement of the dihadron asymmetry on a proton target at COMPASS was performed using the data collected in the year 2007. The results for $h^{+} h^{-}$pairs as a function of $x, z$ and $M_{i n v}$ were presented in [5]. Recently $h^{+} h^{-}$results have been published [9], where the 2007 data have been combined with the most recent and largest data set on the proton target taken in 2010. In both samples a large asymmetry up to $-10 \%$ in the valence $x$-region is present. As for the $z$ dependence, no specific trend is vis-

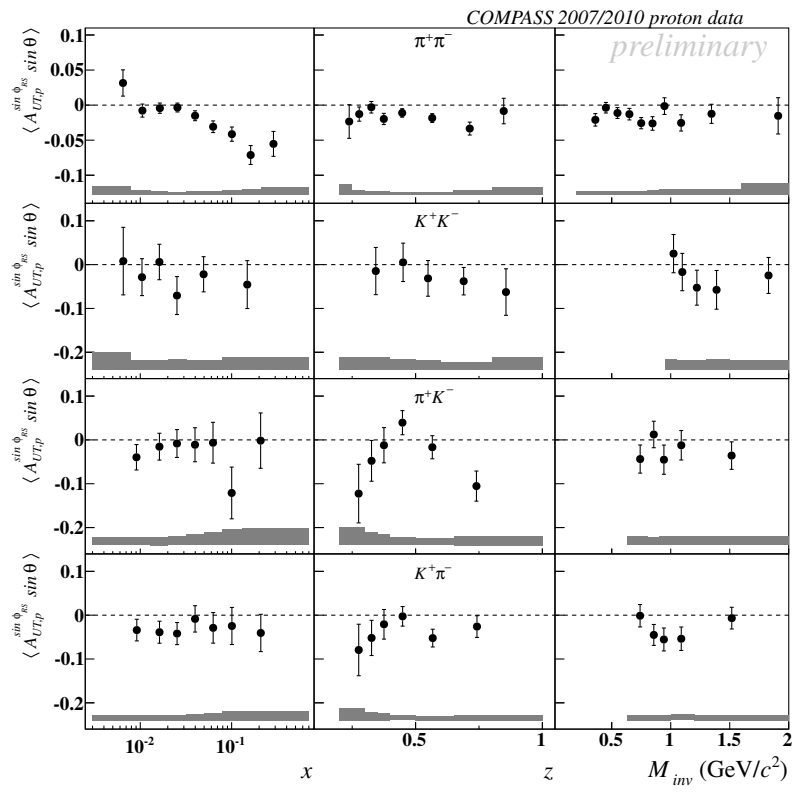

Figure 3. The identified dihadron asymmetries from the combined 2007/2010 proton data [11].

ible, while for the invariant mass a negative signal around the $\rho$ mass of $0.770 \mathrm{GeV} / c^{2}$ is observed and the asymmetry is negative over the whole mass range.

The identified pion-pair asymmetry presented in Fig. 3 shows a clear signal up to $-6 \%$ in $x$, the $z$ dependence is compatible with a constant and for $M_{i n v}$ a pronounced peak around the $\rho$ mass is observed. The asymmetries of the other identified pair combinations $K^{+} K^{-}$and $K^{+} \pi^{-}$give some weak indications of nonzero signals as functions of their invariant masses.

The good agreement of the $\pi^{+} \pi^{-}$asymmetry amplitudes measured by COMPASS with the results obtained by the HERMES experiment [10] and also with the available model predictions [6,7] is discussed in detail in Ref. [11].

\section{Extraction of the Transversity distribution}

The complete sets of dihadron asymmetries obtained from the data taken on the transversely polarized deuteron and proton targets at the COMPASS experiment give the unique possibility to extract the Transversity distributions $x h_{1}^{q}(x)$ of $u$ and $d$ valence quarks separately, without the necessity to evolve results between different kinematic domains. This was done for the first time in a COMPASS $\mathrm{PhD}$ thesis [12], following a method proposed originally for the HERMES proton data [13] and later for the COMPASS proton data [14]. The procedure executed in the following is based on the method by Bacchetta et al. [15] and their previous works, which is referenced therein.

The starting points are the linear combinations $x h_{1, p}\left(x, Q^{2}\right)=x h_{1}^{u}\left(x, Q^{2}\right)-\frac{1}{4} x h_{1}^{d}\left(x, Q^{2}\right)$ and $x h_{1, d}\left(x, Q^{2}\right)=x h_{1}^{u}\left(x, Q^{2}\right)+x h_{1}^{d}\left(x, Q^{2}\right)$, which can be ex- 

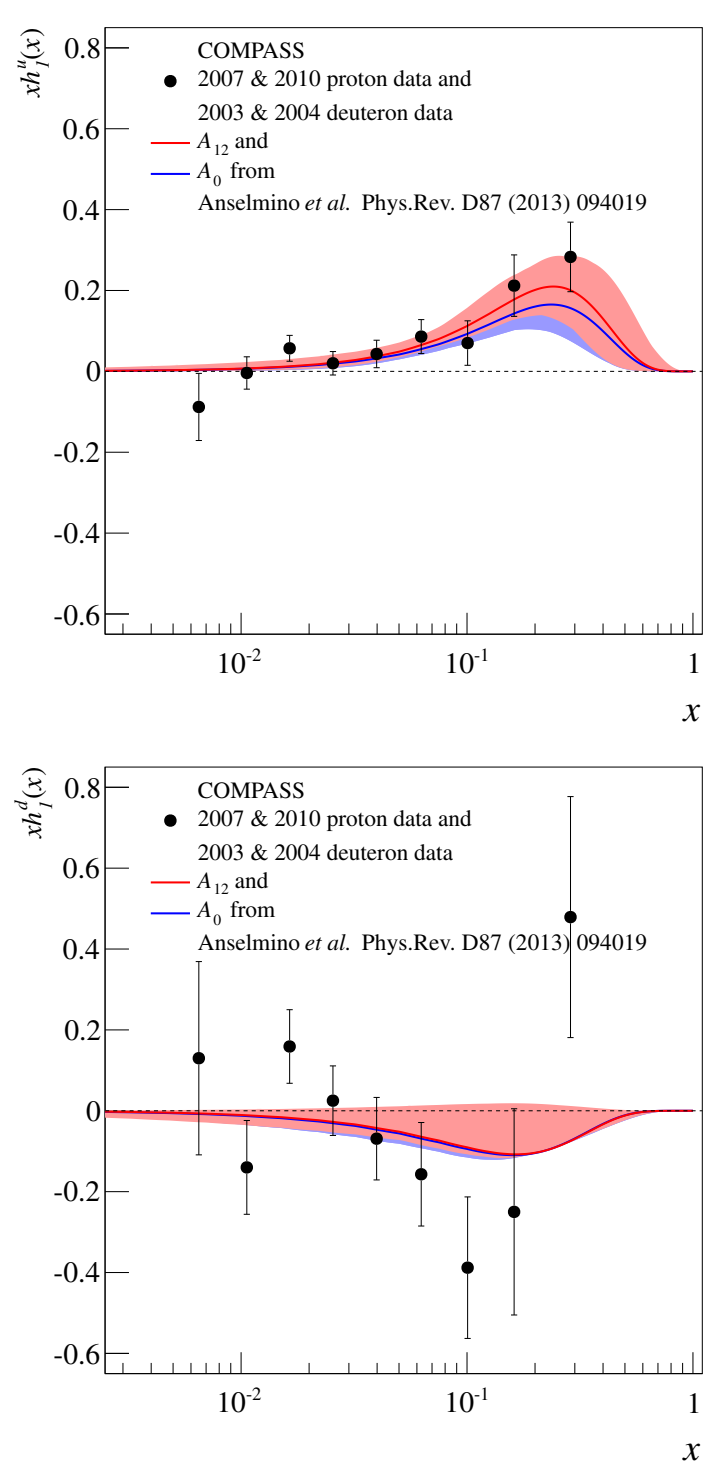

Figure 4. Point-by-point Transversity distributions for $u$ (top panel) and $d$ (bottom panel) valence quarks obtained from this data. They are compared to the corresponding results extracted from the single hadron Collins asymmetries by Anselmino et al. [16] using the $A_{12}$ asymmetry (red curve) and the $A_{0}$ asymmetry (blue curve) from BELLE [17].

tracted from the proton and the deuteron data separately. Using Table 1 of Ref. [15] the bin-by-bin ratios between $x h_{1, p / d}$ and the COMPASS dihadron asymmetries of $h^{+} h^{-}$ pairs used as an input for their extraction are calculated. These ratios contain the information on the unpolarized process as well as on the polarized DiFFs $H_{1}^{\varangle q}$. They can be used as conversion factors in order to calculate the point-by-point amplitudes of the Transversity distribution with the new $\pi^{+} \pi^{-}$results instead the previously used $h^{+} h^{-}$results [5].

The distributions of $h_{1, p / d}$ are very similar in magnitude to the original asymmetry amplitudes, since the conversion factors are almost constant over the whole $x$ range.

The final $u$ and $d$ valence quark Transversity distributions are obtained by solving the system of equations, namely the two linear combinations. Figure 4 shows the results for $x h_{1}^{u}$ and $x h_{1}^{d}$. A nonzero trend at large $x$ values is evident in both distributions, the four highest $x$ bins being at least $1 \sigma$ away from zero. From the present data, sign changes in the distribution of $x h_{1}^{d}$, for instance at very large $x$, can not be excluded due to the limited statistics. Furthermore the $d$ and $u$ quark Transversity PDFs are of opposite signs and the strength of their signals is compatible within the uncertainties. The obtained Transversity signals are also well compatible with the extraction by Anselmino et al. [16] using single hadron Collins asymmetry results and BELLE $e^{+} e^{-}$data [17].

\section{Dependence of the hadron-pair asymmetry on $\Delta \Phi$}

The observation of an almost equal shape and strength of the single hadron Collins asymmetry of $h^{+}$and the mirrored $h^{-}$asymmetry in comparison with the hadronpair $h^{+} h^{-}$asymmetry has recently been pointed out by the COMPASS experiment $[9,18,19]$. Therein also a "Collins"-like hadron-pair angle $\left(\phi_{2 h}+\phi_{S}-\pi\right)$ is introduced, where $\phi_{2 h}$ is simply the arithmetic mean of the azimuthal angles of the two hadrons (after adding a $\pi$ phase to the angle of the negative hadron). The asymmetries calculated using this alternative approach are indistinguishable from the ones obtained using the standard approach. Further efforts have been made to investigate the interplay between the Collins and hadron-pair asymmetry. Among them is the evaluation of the asymmetry as a function of the difference between the azimuthal angle of the positive and the negative hadron, the so-called $\Delta \Phi$ angle.

Figure 5 shows the results obtained (black dots) for the dihadron asymmetry in comparison with the single hadron Collins asymmetry of positively and negatively charged hadrons as a function of $\Delta \Phi$. A clear mirror symmetry is observed, analogous to the one one observes when plotting the three asymmetries as a function of $x$. The asymmetries are compatible to zero when $\Delta \Phi$ is small, and increase steadily as $\Delta \Phi$ grows.

This situation is expected in the framework of the string fragmentation model where the asymmetry is stronger for back-to-back emitted hadrons. Since the difference of the azimuthal angles of the two hadrons is related to the opening angle of the pair, it is interesting to look at the invariant mass distribution of the two hadrons vs. $\Delta \Phi$. Figure 6 shows the invariant mass distribution of $h^{+} h^{-}$pairs for different values of $\Delta \Phi$ in the six bins as used in Fig. 5. Pairs with low invariant mass correspond to small values of $\Delta \Phi$, while for large $\Delta \Phi$ values a clear peak in the number of pairs around $0.8 \mathrm{GeV} / c^{2}$ is observed.

Therefore there is internal consistency between the fact that the hadron-pair asymmetry goes to zero as a function of $\Delta \Phi$ and as a function of the invariant mass. A more detailed and more general study on this promising approach to explain this striking similarity between Collins and Hadron-pair asymmetries is currently being carried out. 


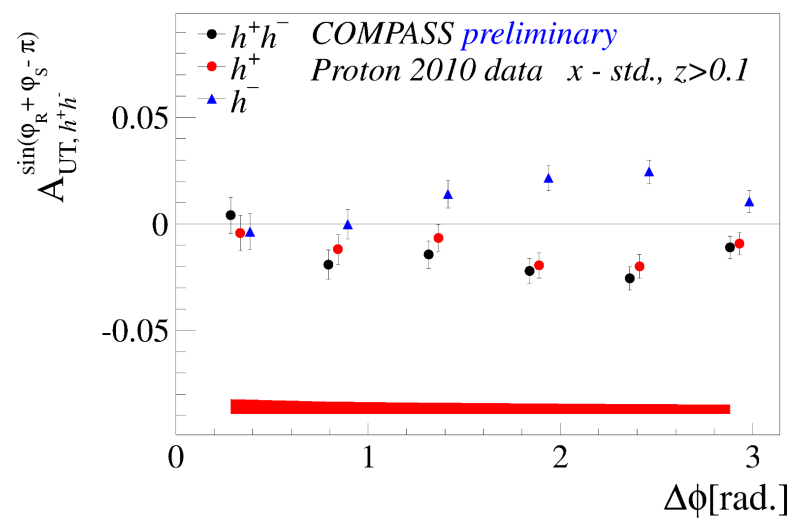

Figure 5. The all hadron-pair $h^{+} h^{-}$asymmetries from the 2010 proton data (black dots) as a function of the the difference of the azimuthal angles of the individual hadrons $\Delta \Phi$ in comparison with the single hadron Collins asymmetry of positively (red dots) and negatively (black dots) charged hadrons as a function of their azimuthal angles.

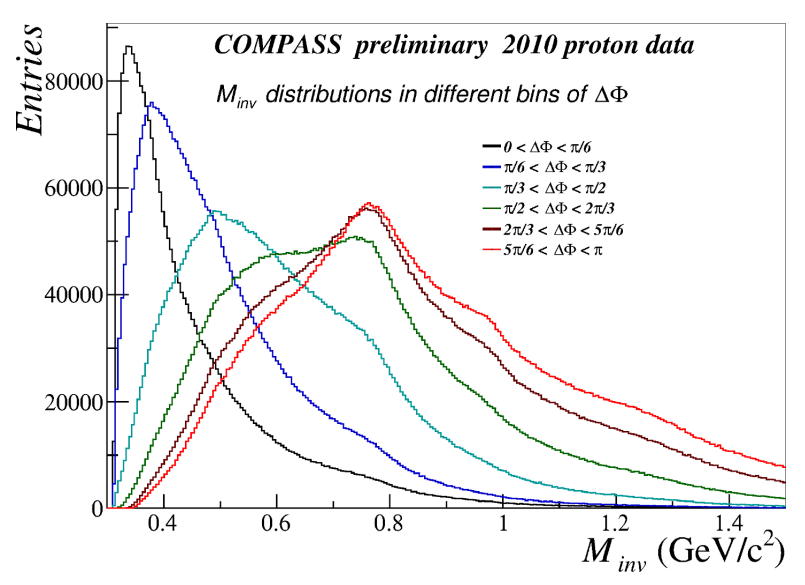

Figure 6. The invariant mass distribution of $h^{+} h^{-}$pairs in six bins of $\Delta \Phi$.

\section{References}

[1] X. Artru and J. C. Collins, Eur. Phys. J. C69, 277 (1996)

[2] A. Bacchetta and M. Radici, Phys. Rev. D67, 094002 (2003)

[3] C. Adolph et al. (COMPASS Collaboration), Phys. Lett. B717, 376 (2012)

[4] M. Alekseev et al. (COMPASS Collaboration), Phys. Lett. B673 127 (2009)

[5] C. Adolph et al. (COMPASS Collaboration), Phys. Lett. B713 10 (2012)

[6] A. Bacchetta and M. Radici, Phys. Rev.D74 114007 (2006)

[7] J. She, Y. Huang, V. Barone, B.-Q. Ma, Phys. Rev. D77 014035 (2008)

[8] C. Braun (COMPASS Collaboration), in proceedings of DIS2014, PoS S203 (2014)

[9] C. Adolph et al. (COMPASS Collaboration), Phys.Lett. B736 124 (2014)

[10] A. Airapetian et al. (HERMES Collaboration), J. High Energ. Phys. 06066 (2008)

[11] C. Braun (COMPASS Collaboration), in proceedings of DIS2013, PoS S232 (2013)

[12] C. Elia, PhD Thesis (2012), University of Trieste

[13] A. Courtoy, A. Bacchetta and M. Radici, Phys. Rev. Lett. 107012001 (2011)

[14] A. Courtoy, A. Bacchetta and M. Radici, PoS QNP2012 042 (2012) arXiv:hep-ph/1206.1836

[15] A. Courtoy, A. Bacchetta and M. Radici, J. High Energ. Phys. 1303119 (2013)

[16] M. Anselmino et al., Phys. Rev. D87 094019 (2013)

[17] A. Vossen, et al. (BELLE Collaboration), Phys. Rev. Lett. 107072004 (2011)

[18] F. Bradamante et al. (COMPASS Collaboration), Talk at International workshop on the Structure of $\mathrm{Nu}$ cleons and Nuclei, Como, Italy (2013)

[19] F. Bradamante et al. (COMPASS Collaboration), Talk at XV Workshop on High Energy Spin Physics, Dubna, Russia (2013), arXiv:1401.6405 [hep-ex] 